\title{
Author Correction: Menin enhances c-Myc- mediated transcription to promote cancer progression
}

Gongwei Wu, Mengqiu Yuan, Shengqi Shen, Xiaoyu Ma, Jingwen Fang, Lianbang Zhu, Linchong Sun, Zhaoji Liu, Xiaoping He, De Huang, Tingting Li, Chenchen Li, Jun Wu, Xin Hu, Zhaoyong Li, Libing Song, Kun Qu, Huafeng Zhang \& Ping Gao

Nature Communications 8:15278 doi: 10.1038/ncomms15278 (2017); Published 5 May 2017; Updated 30 Mar 2018

The originally published version of this Article contained errors in Fig. 6. In panel 1, the Nile Red and Merge images of cells treated with shMYC were inadvertently duplicated from the equivalent images of cells treated with MEN1-sh1 and MEN-sh2 in panel n of the same figure. These errors have now been corrected in the PDF and HTML versions of the Article. For comparison, the original, incorrect version of Figure 61 is presented below as Fig. 1.

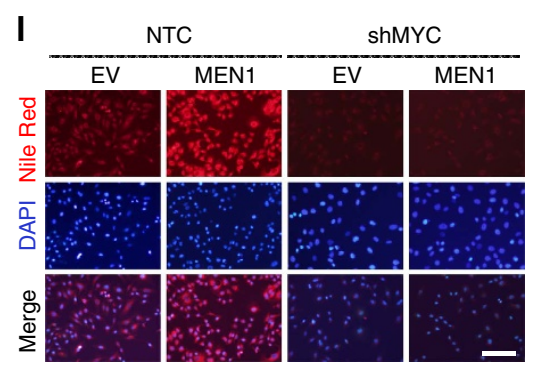

Figure 1

Open Access This article is licensed under a Creative Commons Attribution 4.0 International License, which permits use, sharing, adaptation, distribution and reproduction in any medium or format, as long as you give appropriate credit to the original author(s) and the source, provide a link to the Creative Commons

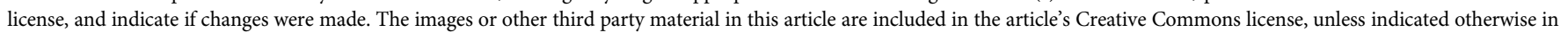

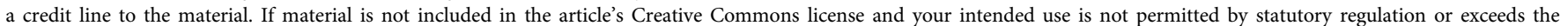
permitted use, you will need to obtain permission directly from the copyright holder. To view a copy of this license, visit http://creativecommons.org/licenses/by/4.0/
\end{abstract}

(C) The Author(s) 2018 